\title{
Fuzzy Filtering for Two Dimensional Images of Mechanical Parts
}

\author{
استخدام الفازى في تثقيه صور الأجز اء المبكانبكية الثنانية الأبعاد
}

\section{Dr. Abdallah A. Alshnnaway , Dr. Ayman A. Aly**}

*Assistant Professor. Design and Prod. Eng. Dept. Tanta University,

**Assistant Professor, Mechatronics Sections, Assiut University. 71516, Egypt

Email: abd_alshennawy@yahoo.com and ayman_aly@yahoo.com

$$
\text { الملخص العربي: }
$$

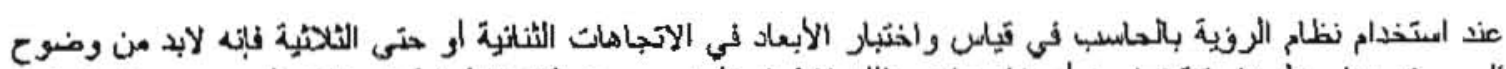

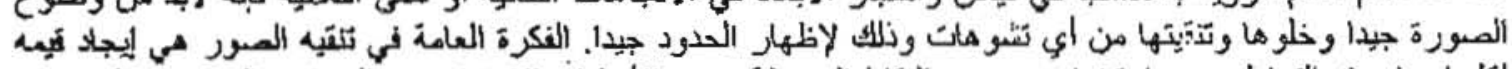

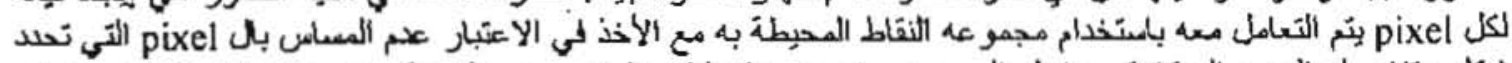

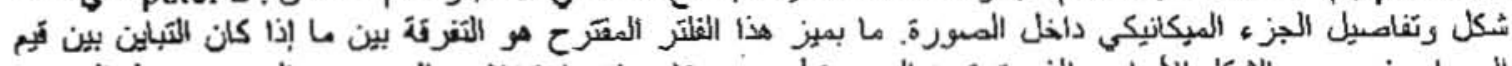

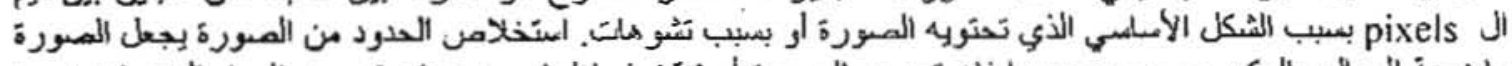

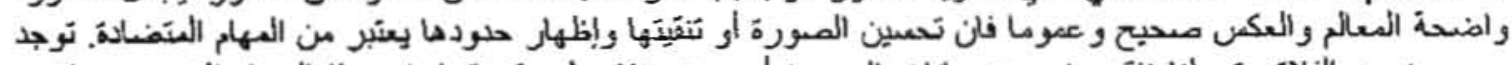

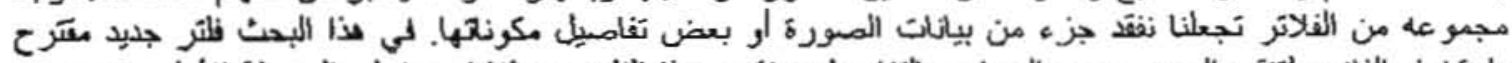

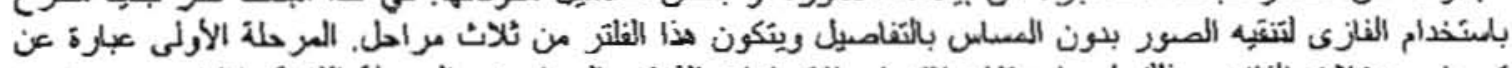

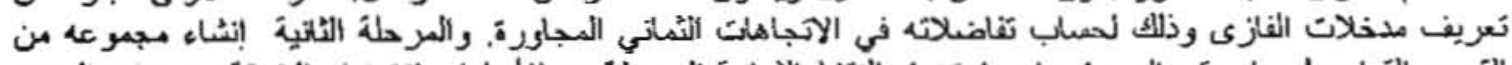

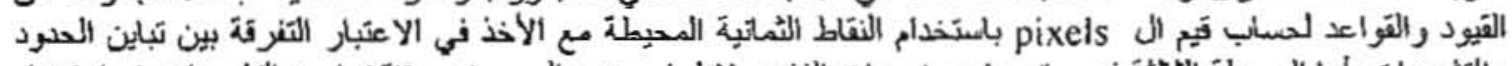

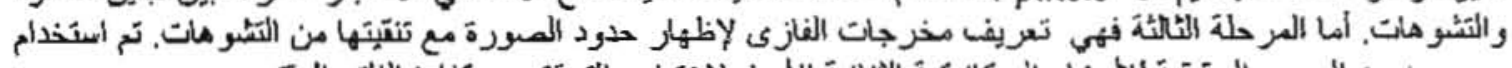

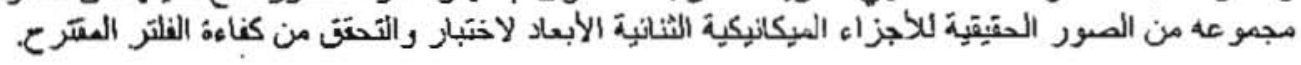

\section{Abstract:}

The general idea behind the filter is to average a pixel using other pixel values from its neighborhood, but simultaneously to take care of important image structures such as edges. The main concern of the proposed filter is to distinguish between any varialions of the captured digital image due to noise and due to image structure.

The edges give the image the appearance depth and sharpness. A loss of edges makts the image appear blurred or unfocused. However, noise smoothing and edge enhancement are traditionally conflicting tasks. Since most noise filtering behaves like a low pass filter, the blurring of edges and loss of detail seems a natural consequence. Tecliniques to remedy this inlicrent conflict often encompass generation of new noise due to enhancement.

In this work a new fuzzy filter is presented for the noise reduction of inages corrupted with additive noise. The filter consists of three stages. (1) Define fuzzy sets in the input space to computes a fuzzy derivative for eight different directions (2) construct a set of IF-THEN rules by to perform fuzzy sinoothing according to contributions of neighboring pixel values and (3) define fuzzy sets in the output space to get the filtered and edged image.

Experimental results are obtained to slow the feasibility of the proposed approach with two dimensional objects.

Keywords: Additive noise, edge preserving filtering, fuzzy image filtering, noise reduction, two dimensional mechanical images. 


\section{2 Abdallah A. Alshnnaway and Ayman A. Aly}

\section{INTRODUCTION}

In the presence of noise, image filtering is an important pre-processing step followed by other tasks such as object recognition, edge detection, feature extraction, pattern recognition and measurement of the features of the images. Generally, linear averaging filters have the ability to remove additive Gaussian noise, but are ineffective against impulse noises (pepper and salt noise).

Noise filtering is an important part of processing and restoring a real image sequence [1]. The noise statistics are usually unknown and differs from one application to another. The more common approach in modeling the noise is to assume that it has Gaussian distribution in addition to some low percentage of additive impulsive noise [2]. The filtering involves the removal or reduction of impulsive noise along with the Gaussian noise while preserving or enhancing edges [3].

Different methods have been proposed in the literature to address this issue. The most effective approaches are of nonlinear and adaptive nature $[1,2]$. Depending on the noise type, we are required to apply the optimal choice of filters to obtain the best output for a particular pixel.

The application of fuzzy techniques in image processing is a promising research field. Fuzzy techniques have already been applied in several domains of image processing (e.g., filtering, interpolation and morphology [3-5]), and have numerous practical applications (e.g., in industrial and medical image processing $[5,6])$.

A robust image enhancement technique was proposed by Dimitri Van De Ville, ...etc, [7] in order to remove impulse noise, smooth out non-impulse noise and preserve edges and other salient structures. There are three different fuzzy filters, one for each task. Their outputs are combined depending on the value of a local feature, which evaluates the gray-level and spatial differences between the central pixel and its neighbors.

Another several fuzzy filters for noise reduction have been developed, e.g., the well-known FIRE-filter from $[8,9]$, the weighted fuzzy mean filter from [10], the iterative fuzzy control based filter from [II]., and the oadaptive fizzy filter proposed by K. Arakawa [12] .

In this paper a nonlinear fuzzy filter, is based on applying local grouping of pixels within a $2-D$ moving window, such as a square mask, over the 2-D input image. The pixels inside the window are ranked according to their gray intensity values. The center pixel is classified to the local groups by using a fuzzy classifier. A pixel that is near an edge will ideally bc combined with the neighboring pixels that lie on the same side of the edge. This requires some form of morphological classification of the local pixels. This algorithm can utilize different morphological structure to capture different patterns and edge characteristics in an image.

The paper is organized as follows. Section II describes vision system III describes the proposed fuzzy filter system, Section IV describes the results and finally Section V reports the conclusions.

\section{VISION SYSTEM}

Due to the rapid progress in the modern manufacturing technology, reliable Automated Visual Systems that offer high speed in conjunction with flexible design are becoming a must. As stated above, this work is an introductory study to apply the computer vision techniques to the field of treatment of the two dimensional digital images. The proposed vision system is based on a matrix CCD-Camera as an image acquisition module and a PC computer, see Fig.1. The captured color image is converted to gray image then the proposed algorithms are applied. 


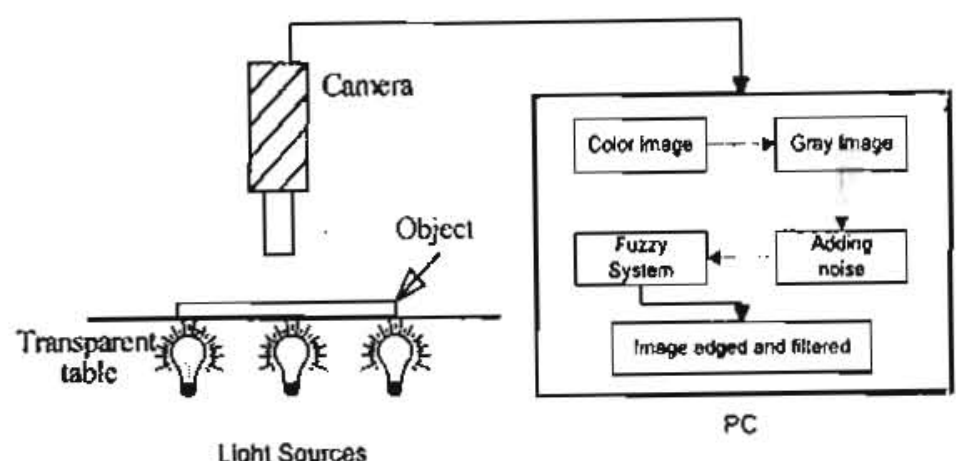

Fig.1 Vision System

\section{FUZZY FILTER}

Image and noise are usually produced at the same time. Pure noise can be manufactured with a computer algorithm. It was then added to a numerical photograph, to simulate a noisy imagc. Although the image is really may be distorted, there is no real loss in visibility. This explains why researchers in image processing believed for a long time that removing noise would not be difficult. However, it is indeed difficuit to separate noise from small image details.

The technological challenge is to remove noise and only noise. Noise reduction in digital images is related to several technological challenges. Fuzzy logic is relatively young theory. Major advantage of this theory is that it allows the natural description, in linguistic terms, of problems that should be solved rather than in terms of relationships between precise numerical values. This advantage, dealing with the complicated systems in simple way, is the main reason why fuzzy logic theory is widely applied in technique.

It is well known that there exist no universal filter and edge detectors which could be applied to a digital image to both identify and track feature outlines with equal efficiency. Instead, we typically have operators which excel in one of these tasks, while performing poorly the other. The combination of operators in complex strategies allows us to overcome this problem. The proposed filter is depends of the design fuzzy system rules as described in the Fig. 2 where $\mathbf{w}$ and $\mathbf{b}$ are means white and black gray level pixels according to the definition of the membership functions see fig.2. The inference rules is depends on the weights of the eight neighbours grey level pixels, if the neighbour's weights are degrees of blacks or degrees of whites. The numbers of rules are over the sixtieth rules. The powerful of these rules is the ability of filtered the image and at the same time extracted the all edges in the processed image. The condition of each pixel is decided by using the floating square mask $3 \times 3$ which can be scanning the all greys of the proposed image. In this situation. some of the desired rules are explained. The rules are dealing with the values of the vertical and horizontal direction grays around the checked or centered pixel $(i, j)$ of the mask, if the all grays around the checked pixel are blacks and the checked gray is white then the checked gray is black. If the grays represented in two directional are blacks and the checked gray is white then the checked gray is black. Also, if the grays represented in two directional are whites and the cliecked gray is black then the checked gray is white. For another examples of the desired rules, as shown in Fig.2, the first rule tell that if $P^{\prime}((i-1, j+1)$ as described in the floating mask in fig.2) is black, $\mathrm{P}^{2}$ is white, $\mathrm{P}^{3}$ is black, $\mathrm{P}^{4}$ is black, $\mathrm{P}^{5}$ is black, $\mathrm{P}^{6}$ is black, $\mathrm{P}^{7}$ is black. $\mathrm{P}^{8}$ is black and $\mathrm{P}^{9}$ is black then from the fuzzy proposed system the $\mathrm{P}^{9}$ is black or the 
checked pixel become black the second rule tell that if $\mathrm{P}^{1}$ is white, $\mathrm{P}^{2}$ is white, $\mathrm{P}^{3}$ is black, $\mathrm{P}^{4}$ is white, $\mathrm{P}^{5}$ is white, $\mathrm{P}^{6}$ is. white,
$\mathrm{P}^{7}$ is white, $\mathrm{P}^{8}$ is white and $\mathrm{P}^{9}$ is white then from the fuzzy inference system the $\mathrm{P}^{9}$ is edge or the checked pixel become edge.
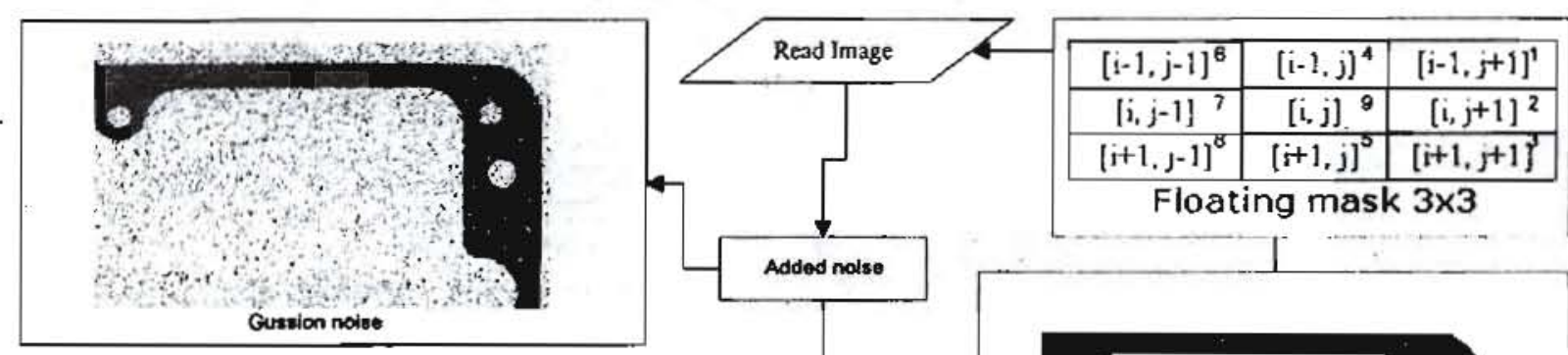

Floating mask $3 \times 3$
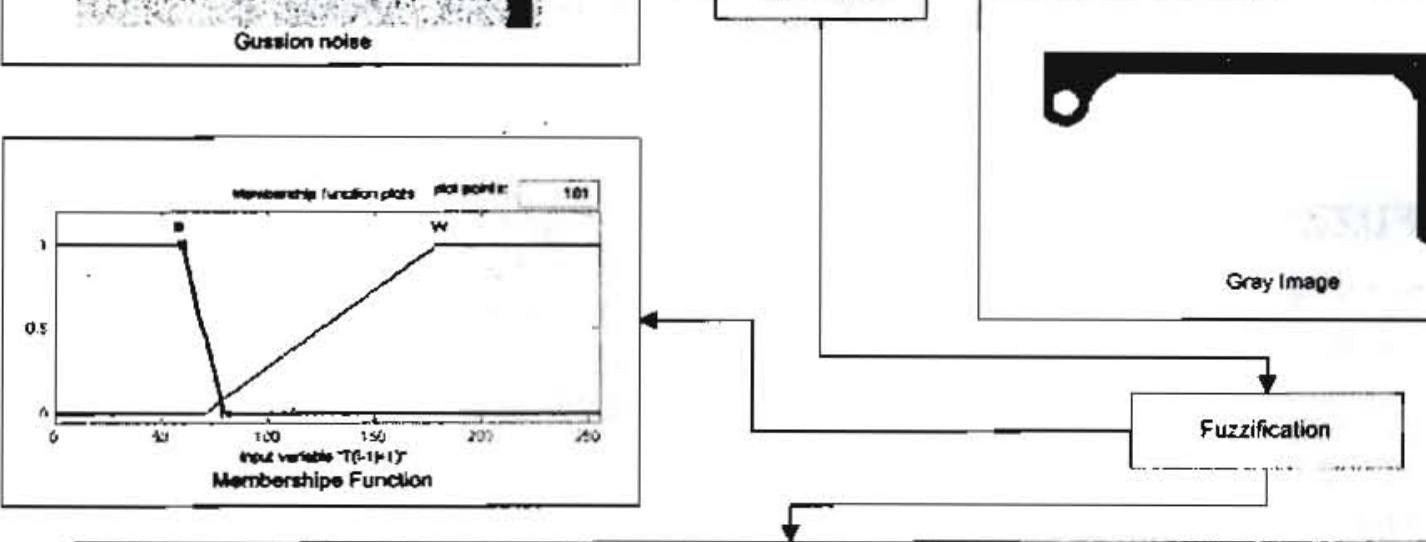

Rule 1: If $[T(i-1, f+1\}$ is $b]$ and $[T(j, j+1)$ is $w\}$ and $[T(l+1, j+1)$ is $b)$ and $[T(i-1, j)$ is b] and $[T(i+1, j)$ is $b]$ and $[T\{i-1, j-1)$ is $b]$ and $[T(b j-1)$ is $b]$ and $[T(i+1, j-1)$ is $b]$ and $[T(i, j)$ is b] Then the checked pixel $T(i, j)$ is black

Rule 2: $I f[T(i-1, j+1)$ is $w]$ and $[T(i j+1)$ is $w]$ and $[T(i+1, j+1)$ is b] and $[T(i-1, j)$ is $w]$ and $[T(i+1, j)$ is $w]$ and $[T(i-1, j-1\}$ is $w]$ and $[T(I,-1)$ is $w]$ and $[T(I+1, f-1)$ is $w]$ and $[T(i, j)$ is $w]$ Then the checked pixel $T(i, j)$ is Edge

Rule $n-1$ : If $[T(-1, j+1)$ is b] and $[T(i, j+1)$ is $b]$ and $[T(i+1, j+1)$ is $b]$ and $[T(1-1, j)$ is b] and $[T(i+1, j)$ is $b]$ and $[T\langle i-1, j-1\rangle$ is $w]$ and $[T(1, j-1)$ is $w]$ and $[T(I+1 j-1)$ is $w]$ and $[T(I, j)$ is $w]$ Then the checked pixel $T(L, j)$ is White

Rule $n$ : If $[T(i-1, j+1)$ is b] and $[T(1, j+1)$ is $w]$ and $[T(i+1, j+1)$ is $w]$ and $[T(i-1, j)$ is b] and $[T(i+1, j)$ is $w]$ and $[T(i-1, j-1)$ is $b]$ and $[T(I J-1)$ is b] and $[T(I+1,-1)$ is b] and [T(IJ) is b] Then the checked pixel $T(i, j)$ is black

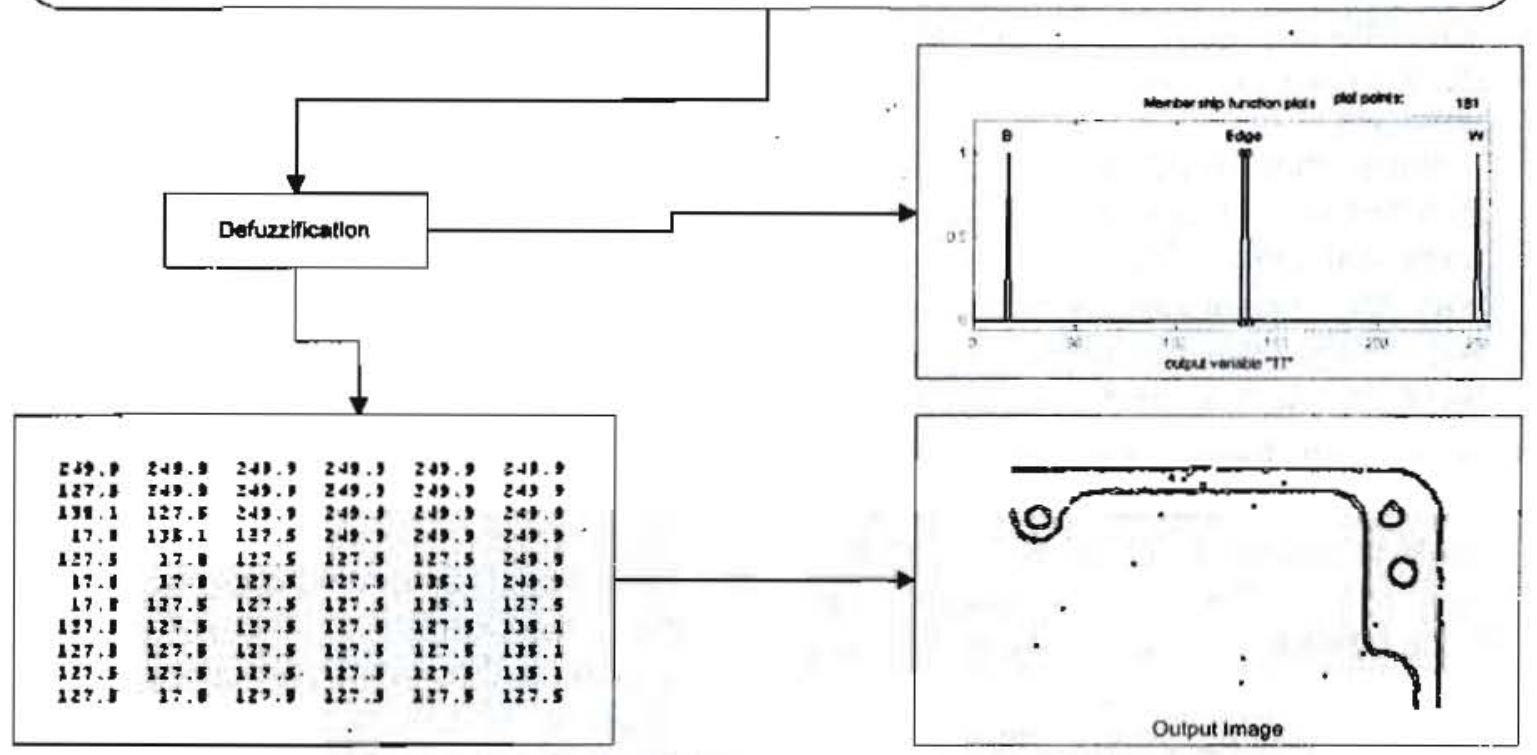

Fig.2. The Proposed Fuzzy Filter and Edge Detection Flow chart 


\subsection{The Flow of the Proposed Fuzzy System}

The main procedure of this proposed filter is preceded as shown in Fig.2:

1. Added the Salt and Paper noise or Gaussian noise to its gray level image. These noises through the MATLAB standard functions.

2. Implementing the fuzzy membership functions of the input noise image (fuzzification) [6].

3. Design the fuzzy system rules as described in the previous section.

4. At the same time another groups of the rules are derived to extract the edges of the mechanical objects in the image as described in the previous section [6].

5. Implementing the output membership functions as shown in the Fig. 2 (defuzzification).

6. The resulted matrix is represented the output values of the proposed fuzzy filter of the gray level input pixels. [6].

7. The resulted image is the filtered image from the noise and extracted the edges of the features of the image.

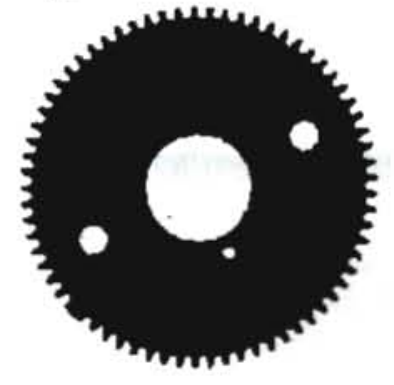

a

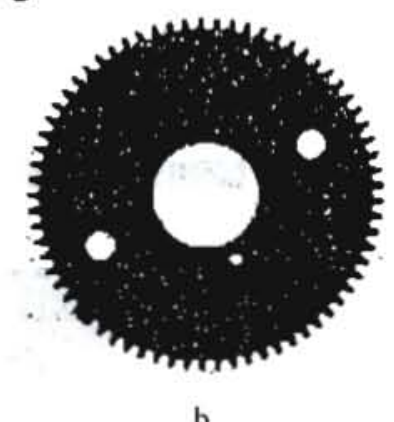

b

\section{EXPERIMENTAL RESULTS}

The proposed filter is applied to grayscale test images (8-bit, $\mathrm{L}=255$ ), after adding salt and pepper noise of different levels. All images have noise, even if it is usually less conspicuous. Noise is due to the actuations of the light and the photo electronic activity of the sensors. Our own perception also generates noise, which one notices when opening eyes in darkness, or by simply closing the eyes.

We like to show a practical application of the proposed fuzzy algorithm. Fig.3.a shows the original gear image at the left side and the corrupted one with salt and pepper noise at Fig.3.b. For these noise levels our filter is applied using inference fuzzy technique which produces Fig.3.c. To verify the result of the proposed fuzzy filter, we added another type of the noise (Gaussian) to the original image at Fig.3.d. and filtered image shown in Fig.3.e. The proposed filter removed the tested noises and in the same time kept the image edges and its original details.
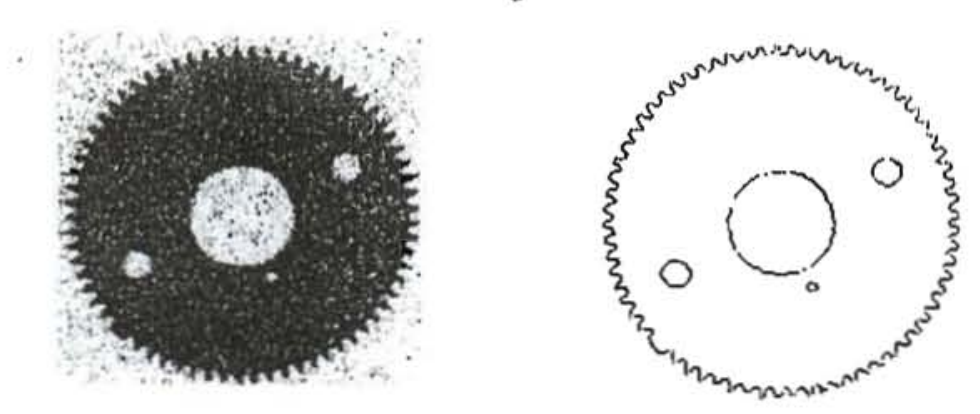

d

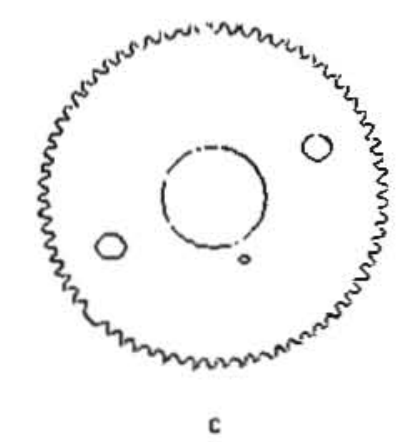

e

Figure 3.a The original gear image, b. The added salt and pepper noise with the original image, c. The filtered image by the proposed filter with salt and pepper noise, $d$. The added Gaussian noise with the original image and $e$. The filtered image by the proposed filter with Gaussian noise 


\section{6 Abdallah A. Alshnnaway and Ayman A. Aly}

Finally, to ensure the robustness of the proposed filter, another real image was tested by adding the salt and pepper noise in Fig.4.b. By filtration this image, a clcar and clean edge is appeared at Fig.4.c. At Fig.4.d.the Gaussian noise was added to the same image and its filtered result was presented at Fig.4.e.
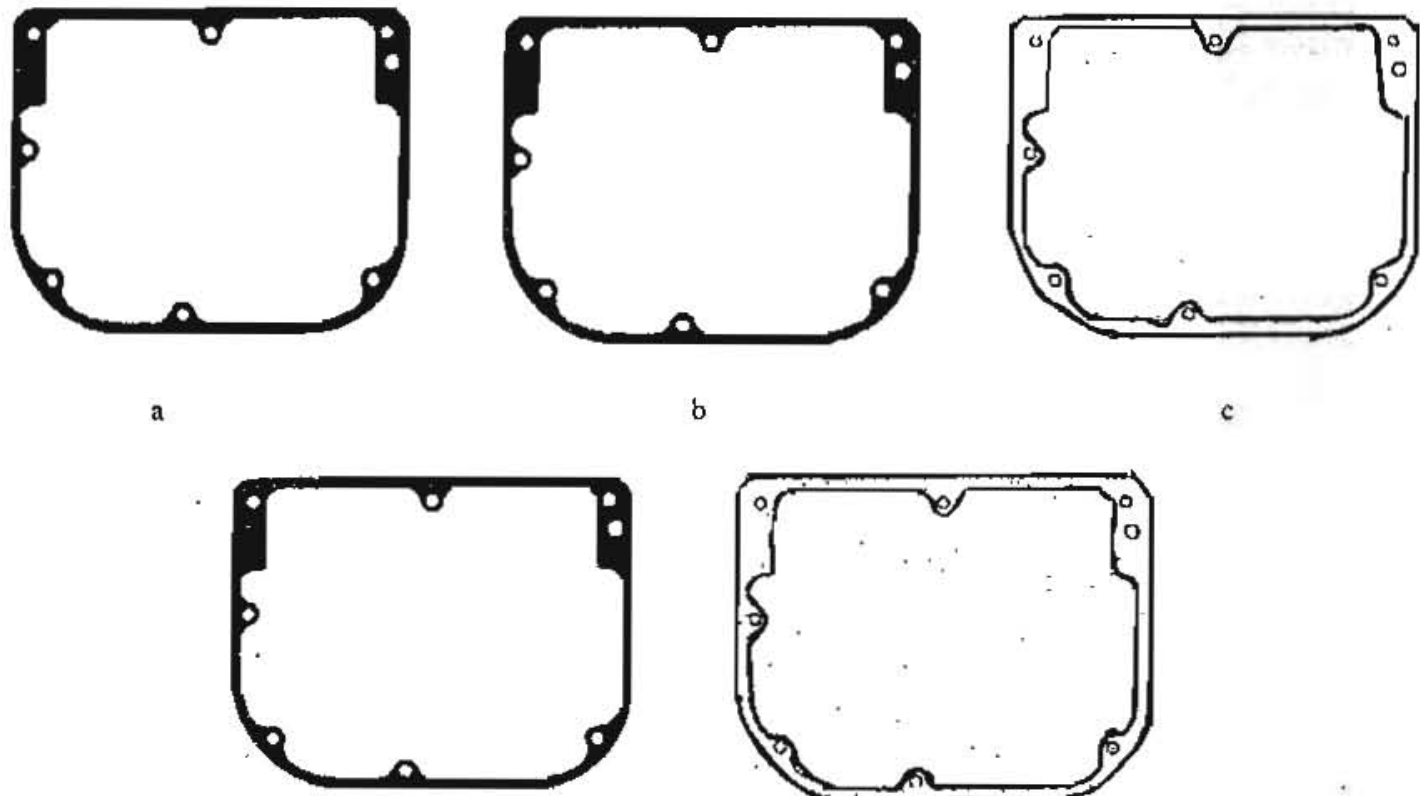

d
In particular, this images restoration scheme could be used to enhance captured images. Of course, since the original image is already corrupted by noise, it is not possible to obtain a numerical measure which indicates how "good" the image is. c

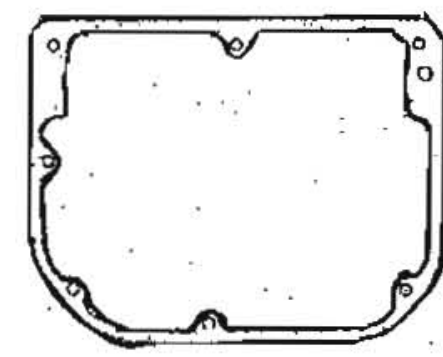

e

Figure 4.a The original seal image, b. The added salt and pepper noise with the original image, c.

The filtered image by the proposed filter with salt and pepper noise, $d$. The added Gaussian noise with the original image and $e$. The filtered image by the proposed filter with Gaussian

\section{CONCLUSION}

This paper proposed a new fuzzy filter and edge detector technique for additive noise reduction and in the same time detects the captured image edges. Its main feature is that it distinguishes between local variations due to noise and image structures, using fuzzy inference estimation. Fuzzy rules are fired to consider every direction around the processed pixel. Experimental results show the feasibility of the new filter, and a simple detection and filtration mechanism. Although it's relative simplicity and the straightforward implementation of the fuzzy operators, the fuzzy filter is able to compete with state-of-the-art filter and detectors techniques for noise reduction. noise

Finally, the fuzzy policy scheme is sufficiently simple to enable fast hardware implementations.

\section{REFERENCES}

[1] Raghu Krishnapuram, Hichem Frigui, and Olfa Nasraoui," Fuzzy and Possibilistic Shell Clustering Algorithms and Their Application to Boundary Detection and Surface Approximation", IEEE Transactions on Fuzzy Systems, Vol. 3, No. 1, February 1095.

[2] Nedeljkovic, "Image Classification Based On Fuzzy Logic" The International Archives of The Photogrammetry, Remote Sensing And 
Mansoura Engineering Journal, (MEJ), Vol. 33, No. 3, September 2008.

M. 7

Spatial Information Sciences, Vol. 34, Part XXX.

[3] William A. Gowan, "Optical Character Recognition Using Fuzzy Logic", Order this document by: AN1220/D Semiconductor Motorola Application Note.

[4] B.-G. Hu, R. G. Gosine, L. X. Cao, and C. W. de Silva, "Application of a Fuzzy Classification Technique in Computer Grading of Fish Products ", IEEE Transactions on Fuzzy Systems, Vol. 6, No. 1, February 1998.

[5] S. Singh and A. Amin. Fuzzy Recognition of Chinese Characters, Proc. Irish Machine Vision and Image Processing Conference (IMVIP'99), Dublin, (8-9 September, 1999).

[6] Abdallah A. Alshnnaway, Ayman A. Aly, "Fuzzy Logic Technique Applied to Extract Edge Detection in Digital Images For Two Dimensional Objects", International conference in Production Engineering, METIP 4, 15-17 December 2006.

[7] Dimitri Van De Ville, Mike Nachtegael, Dietrich Van der Weken, Etienne E. Kerre, "Noise Reduction by Fuzzy Image Filtering", IEEE Transactions on Fuzzy Systems, Vol. 11, No. 4, August 2003.

[8] Antoni Buades, Bartomeu Coll and Jean-Michel Morel, A Review of lmage Denoising Algorithms, With a New One to appear in Multiscale Modelling and Simulation," 2005. No French Patent application registered on May 5, 2004. (Prepublication avalaible at https://www.cmla.ens-cachan.fr).

[9] F. Russo, "Fire operators for image processing", Fuzzy Sets System., vol. 103, no. 2, pp. 265-275, 1999.

[10] C.-S. Lee, Y.-H. Kuo, and P.-T. Yu, "Weighted fuzzy mean filters for image processing," Fuzzy Sets System., no. 89, pp. 157-180, 1997.
[11] C.-S. Lee and Y.-H. Kuo, "Fuzzy Techniques in Image Processing", New York: Springer-Verlag, 2000, vol. 52. Studies in Fuzziness and Soft Computing, ch. Adaptive fuzzy filter and its application to image enhancement, pp. 172-193.

[12] K. Arakawa, "Median filter based on fuzzy rules and its application to image restoration," Fuzzy Sets System., pp. 3-13, 1996. 